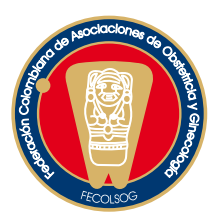

REPORTE DE CASO

\title{
CARDIOMIOPATÍA PERIPARTO: REPORTE DE UN CASO Y REVISIÓN DE LA LITERATURA*
}

\section{Peripartum cardliomyopathy: a case report and literature review}

\author{
Alejandro Hernández-Guzmán, M.D.**, Albaro José Nieto-Calvache, M.D.**, \\ Virna Medina-Palmezano, M.D.***, Julián Delgado-Gutiérrez, M.D., MSc**** \\ Recibido: junio 4/08 - Aceptado: julio 31/09
}

\section{RESUMEN}

Objetivo: realizar una revisión de la literatura acerca de los aspectos más relevantes del manejo de la cardiomiopatía periparto (CMPP) en la unidad de alta dependencia obstétrica.

Presentación del caso: presentamos un caso clínico con diagnóstico de CMPP al cuarto día posparto. El ecocardiograma identificó un compromiso de la fracción de eyección. El tratamiento intensivo y adecuado permitió la supervivencia y la recuperación de la función ventricular sistólica izquierda.

Discusión: la CMPP es una enfermedad cardíaca rara que ocurre durante el período posparto, la cual ha sido reconocida como una entidad distinta al infarto de miocardio cuyos signos y síntomas tempranos de falla cardíaca pueden estar ocultos por las modificaciones normales del embarazo. La evidencia indica que la tasa de letalidad varía entre 9\% y 56\% con la mayoría de las muertes ocurridas dentro de los tres primeros meses posparto.

* $\quad$ Presentado en el Congreso Colombiano de Perinatología. Medellín (Colombia) 2007. Póster. Pie de página: cardiomiopatía periparto.

** Residente de Ginecología y Obstetricia. Universidad del Valle. Hospital Universitario del Valle. Departamento de Ginecología y Obstetricia. Dirección: calle 5 No. 36-05. (Cali, Colombia). Correo electrónico: alherguz01@hotmail.com

*** Especialista en Ginecología y Obstetricia. Universidad del Valle. Profesora Contratista, Departamento de Ginecología y Obstetricia. Universidad del Valle (Cali, Colombia).

**** MSc en Obstetricia. Universidad Autónoma de Barcelona (España). Especialista en Ginecología y Obstetricia. Universidad del Valle. Profesor Asistente, Departamento de Ginecología y Obstetricia. Universidad del Valle (Cali, Colombia).
Su diagnóstico se basa en la presentación clínica de falla cardíaca congestiva y la evidencia objetiva de disfunción sistólica ventricular izquierda. Por lo tanto, el tratamiento debe incluir la terapia para falla cardíaca, la cual implica la reducción de la precarga, la poscarga y el uso de agentes inotrópicos, diuréticos, vasodilatadores y la digoxina. Igualmente, los inhibidores de la enzima convertidora de angiotensina (IECA) deben ser incluidos en el tratamiento posparto de la CMPP. La finalización del embarazo depende del estado funcional y de la respuesta al tratamiento inicial. Por esto, el obstetra juega un papel fundamental en el abordaje multidisciplinario de las mujeres con esta entidad y debe coordinar los esfuerzos del equipo tratante.

Palabras clave: cardiomiopatía periparto, falla cardíaca, embarazo.

\section{SUMMARY}

Objective: reviewing the relevant literature concerning medical care and management in a peripartum cardiomyopathy (PPCM) high obstetrical dependence unit.

Case report: this article presents a case diagnosed as having PPCM on the 4th day postpartum. An echocardiogram revealed a compromised ejection fraction. Intensive, appropriate treatment led to survival and recovery of left ventricular systolic function. 
Discussion: $\mathrm{PPCM}$ is a rare cardiac disorder which occurs during the peripartum period. It has been recognised as a distinct entity from myocardial infarction. Its exact incidence remains unknown but mortality rate ranges from $9 \%$ to $56 \%$, most deaths being reported during the first three months postpartum. Diagnosis is based upon the clinical presentation of congestive heart failure and objective evidence of left ventricular systolic dysfunction. Treatment should include standard therapy for heart failure, thus implying reduced preload, postload and inotropic, diuretic, vasodilator agents and digoxin use. Angiotensin-converting enzyme inhibitors (ACEI) must be included in CMPP postpartum treatment. The outcome of pregnancy depends on functional state and response to initial treatment. The obstetrician plays a fundamental role in a multidisciplinary approach to females suffering from this entity and must coordinate team efforts.

Key words: peripartum cardiomyopathy, cardiac failure, pregnancy.

\section{INTRODUCCIÓN}

Descrita por primera vez en 1870 por Virchow y Porack, la cardiomiopatía periparto (CMPP) es una enfermedad cardíaca rara que ocurre durante el período posparto. Sin embargo, solamente hasta 1937 Gouley y colaboradores identificaron la causa de falla cardíaca (FC) como una cardiomiopatía dilatada, la cual ha sido reconocida como una entidad distinta al infarto de miocardio desde entonces. La CMPP corresponde al desarrollo de falla cardíaca en los últimos meses del embarazo y en los primeros 5 meses posparto. ${ }^{1}$ La incidencia exacta es desconocida, y su prevalencia es diferente en cada grupo estudiado: 1/299 recién nacidos vivos (RNV) en Haití, ${ }^{2}$ 1/1.000 RNV en Sudáfrica ${ }^{3}$ y 1/4.000 RNV en EUA. ${ }^{4}$ Actualmente, es considerada la primera causa de mortalidad materna por enfermedad cardíaca (20\%), ${ }^{5}$ y se han descrito como factores de riesgo para CMPP: la edad (más de 30 años), la multiparidad, la obesidad, los trastornos hipertensivos del embarazo, la raza negra, el uso de tocolíticos y el embarazo gemelar. ${ }^{6}$ Esta enfermedad también se ha relacionado con la desnutrición, la ausencia de controles prenatales y la lactancia, ${ }^{7}$ aunque otros estudios no han encontrado dicha asociación. ${ }^{8}$ Igualmente, las zonas tropicales, con calor y humedad se han asociado con la entidad. ${ }^{9}$

Pese a que se han postulado diferentes teorías etiológicas para la CMPP, entre las cuales cabe mencionar: miocarditis, ${ }^{10}$ infecciones por clamidia u otros microorganismos, ${ }^{11-13}$ apoptosis de los cardiomiocitos,${ }^{14}$ autoinmunidad, ${ }^{15}$ deficiencias nutricionales ${ }^{16}$ y costumbres específicas p.ej. el consumo de grandes cantidades de sales como se ha observado en algunas tribus en África, ${ }^{17}$ la causa de la CMPP sigue siendo desconocida, lo que dificulta la implementación de un enfoque terapéutico específico.

A ésto se le suma el hecho de que la entidad presenta los mismos signos y síntomas de la FC al igual que cualquier miocardiopatía dilatada, y debe sospecharse siempre en este escenario, lo cual representa un reto para el diagnóstico por la común ocurrencia de edema, disnea y fatiga en el último mes de embarazo en mujeres sin patología. Por consiguiente, el objetivo de este reporte de caso es brindar al obstetra una revisión de los aspectos relacionados con el diagnóstico y el tratamiento de la CMPP para lograr la adecuada coordinación del caso en el manejo multidisciplinario indispensable en la paciente con esta patología y para no limitar su papel a transferir a la paciente a una unidad de cuidados intensivos.

\section{CASO CLÍNICO}

Paciente de 32 años, $\mathrm{G}_{3} \mathrm{P}_{2} \mathrm{C}_{2} \mathrm{M}_{1}$, sin antecedentes de cardiopatía o de síntomas como disnea o dolor torácico, a quien se le realizó cesárea por feto en situación transversa de 34 semanas y en preeclampsia severa. Durante los 3 días de la primera hospitalización se le administró 6.000 cc de solución salina 
0,9\% vía endovenosa. A los cuatro días de la cesárea, la mujer consultó por presentar disnea progresiva asociada con tos con expectoración hemoptoica que progresó a falla respiratoria, pese a mostrarse asintomática al momento del egreso. A su ingreso, se observó en la paciente un estado normotenso, con roncus generalizados, utilización de músculos accesorios de dos días de evolución e inminencia de falla respiratoria, por lo cual fue necesario su intubación oro traqueal. Inmediatamente, se le tomó una radiografía de tórax revelando cardiomegalia con infiltrados parahiliares bilaterales. Asimismo, se le realizó ecocardiograma transesofágico el cual expuso una fracción de eyección del ventrículo izquierdo (FEVI) de 30\%, ventrículo izquierdo dilatado con hipocinesia global, aurícula izquierda dilatada con presión pulmonar central de $40 \mathrm{~mm} \mathrm{Hg}$ e hipocinesia del ventrículo derecho con dilatación de cavidades derechas. Se consideró el diagnóstico de falla cardíaca y edema pulmonar, por lo cual se inició el manejo con furosemida (20 mg/día), dobutamina $(10 \mu \mathrm{g} / \mathrm{kg} / \mathrm{min})$, carvedilol (25 mg/día), digoxina (0,25 mg/día), enalapril (10 mg/día), espironolactona (25 mg/día), sedación para ventilación mecánica, tromboprofilaxis pulmonar con enoxaparina (40 mg/día), y nutrición enteral total. Ante la presencia de infiltrados bilaterales parahiliares se consideró la posible manifestación de neumonía adquirida en la comunidad versus neumonía nosocomial, por lo cual se comenzó el manejo con clindamicina (2.400 mg/día).

En su tercer día de hospitalización, la paciente presentó en forma súbita la alteración del estado de conciencia con desviación de la mirada hacia la derecha, sialorrea profusa, signos de hipoperfusión y relajación de esfínteres. Después de practicado un electrocardiograma, éste evidenció taquicardia con frecuencia cardíaca de 220 latidos por minuto y complejos angostos $\mathrm{R}-\mathrm{R}$ irregulares, por lo cual se le diagnosticó FV (Fibrilación Ventricular) y se iniciaron maniobras de reanimación cardiopulmonar básica y avanzada y manejo con amiadorona (300 mg), revirtiendo a ritmo sinusal.

Posteriormente, la paciente presentó una buena evolución de los parámetros hemodinámicos y gasométricos, razón por la cual se decidió extubar a los 8 días de hospitalización. Inicialmente, dio muestras de episodios de broncoespasmo que cedieron con la administración de corticosteroides. Tiempo después, al no presentar signos clínicos ni paraclínicos de infección respiratoria, se tomó la decisión de suspender el antibiótico.

Se realizó cateterismo cardíaco de control a los 15 días, con el cual no se evidenciaron hallazgos patológicos en los vasos coronarios ni lesiones valvulares, pero sí se reveló la dilatación del ventrículo izquierdo con fracción de eyección de 40\%. Finalmente, la paciente presentó buena evolución clínica, por lo cual se le dio salida con cita de control por consulta externa de medicina interna (cardiología) y ginecología.

\section{DISCUSIÓN}

Se conoce muy poco acerca de la fisiopatología y del pronóstico de la CMPP. Aún con los avances en el tratamiento médico para la falla cardíaca, esta entidad se asocia con una mortalidad significativa. ${ }^{18}$ Por consiguiente, el conocimiento que el obstetra adquiera de las características semiológicas, de los criterios de diagnóstico, de las intervenciones terapéuticas y de las variables de pronóstico le permitirán el reconocimiento precoz de esta entidad y de su participación activa en el cuidado de la paciente críticamente enferma. ${ }^{19}$

Siempre debe realizarse una valoración clínica detallada en busca de las posibles etiologías y debe considerarse el diagnóstico de la CMPP ante la exclusión de otras patologías, en toda paciente embarazada o en aquella que en los primeros 5 meses posparto manifieste síntomas de falla cardíaca.

Los criterios de diagnóstico incluyen, además del tiempo de presentación, la ausencia de disfunción cardíaca preexistente, la ausencia de 
una causa determinable de cardiomiopatía y más recientemente, la disfunción ventricular sistólica demostrada por principios ecocardiográficos clásicos: fracción de eyección (FE) menor a 45\%, acortamiento fraccional en modo $\mathrm{M}$ menor de $30 \%$ o ambos, y dimensión de fin de diástole mayor de $2,7 \mathrm{~cm} / \mathrm{m}^{2} .^{20-22}$ La evaluación y el seguimiento de la fracción de eyección ventricular son indispensables.

De confirmarse el diagnóstico, se debe iniciar el manejo multidisciplinario cardiovascular intensivo. El manejo de la CMPP consiste en el soporte hemodinámico de la paciente con FC, y la terapia farmacológica está orientada a disminuir la precarga, la poscarga y a mejorar la contractilidad del músculo cardíaco. ${ }^{23}$ Además, el alto riesgo de tromboembolismo hace necesaria la anticoagulación profiláctica con heparina. Por otro lado, la finalización del embarazo en pacientes con CMPP está determinada por el estado funcional y el bienestar fetal y sólo será necesaria precozmente si ocurre alguna falla en la respuesta a la terapia durante el estado de descompensación de la madre. ${ }^{24}$ De otra parte, el pronóstico y el futuro obstétrico dependen de la función ventricular (FEV) medida con los parámetros ecocardiográficos, ${ }^{5,6,25,26}$ pero generalmente la entidad conlleva a una elevada morbimortalidad de manera que el 20\% de las pacientes muere o sobrevive sólo gracias a un trasplante cardíaco ${ }^{27}$ mientras que aproximadamente la mitad de las pacientes se recuperan sin complicaciones. ${ }^{28}$ La enfermedad persistente por más de 6 meses se relaciona con cardiomiopatía irreversible y pobre sobreviva. ${ }^{29}$ El diagnóstico precoz y la iniciación inmediata del tratamiento son esenciales para optimizar el resultado obstétrico.

De otra parte, es importante explicarle a la paciente que desea quedar nuevamente en embarazo que existe un efecto considerable de los embarazos subsecuentes en el resultado materno y fetal. En tres estudios recientes, los cuales analizaron el pronóstico y el futuro obstétrico en la CMPP, Elkayam y sus colegas ${ }^{30}$ evaluaron, como factor pronóstico, las diferencias en la recuperación de la fracción de eyección. Por su lado, Amos y colaboradores ${ }^{31}$ calcularon el mejoramiento de los resultados en pacientes con CMPP bajo tratamiento contemporáneo en el que se les administraron inhibidores de la enzima convertidora de angiotensina y beta bloqueadores. En tercer lugar, Fett y su equipo de trabajo $^{29}$ realizaron un seguimiento durante 5 años a las pacientes con CMPP comparando las diferencias entre las fracciones de eyección. A partir de estos resultados, se llegó a la conclusión de que las pacientes con CMPP tienen una alta probabilidad de presentar falla cardíaca debido a la progresión de los síntomas en embarazos subsecuentes; por lo tanto, se recomienda llevar a cabo un registro que identifique de manera prospectiva a todas las mujeres que están en riesgo. ${ }^{32}$

\section{AGRADECIMIENTOS}

Agradecemos de manera especial al Dr. Álvaro José Caicedo, especialista en Radiodiagnóstico, por su contribución en la interpretación de las ayudas diagnósticas.

\section{REFERENCIAS}

1. Demakis JG, Rahimtoola SH. Peripartum cardiomyopathy. Circulation 1971;44:964-8.

2. Fett JD, Carraway RD, Dowell DL, King ME, Pierre R. Peripartum cardiomyopathy in the Hospital Albert Schweitzer District of Haiti. Am J Obstet Gynecol 2002;186:1005-10.

3. Cenac A, Djibo A. Postpartum cardiac failure in Sudanese-Sahelian Africa: clinical prevalence in western Niger. Am J Trop Med Hyg 1998;58:319-23.

4. Lang CT, King JC. Maternal mortality in the United States. Best Pract Res Clin Obstet Gynaecol 2008;22:517-31.

5. Ray P, Murphy GJ, Shutt LE. Recognition and management of maternal cardiac disease in pregnancy. Br J Anaesth 2004;93:428-39. 
6. Sliwa K, Fett J, Elkayam U. Peripartum cardiomyopathy. Lancet 2006;368:687-93.

7. Arafeh JM, Baird SM. Cardiac disease in pregnancy. Crit Care Nurs Q 2006;29:32-52.

8. Palmer DG. Peripartum cardiomyopathy. J Perinat Neonat Nurs 2006;20:324-32.

9. Murali S, Baldisseri MR. Peripartum cardiomyopathy. Crit Care Med 2005;33:S340-6.

10. Melvin KR, Richardson PJ, Olsen EG, Daly K, Jackson G. Peripartum cardiomyopathy due to myocarditis. N Engl J Med 1982;307:731-4.

11. Cenac A, Djibo A, Sueur JM, Chaigneau C, Orfila J. Chlamydia infection and peripartum dilated cardiomyopathy in Niger. Med Trop 2000;60:137-40.

12. Arnould N, Diemunsch P, Raiga J, Brettes JP. Peripartum dilated cardiomyopathies: is there a correlation with sexually transmitted diseases? Gynecol Obstet Fertil 2002;30:59-63.

13. Bültmann BD, Klingel K, Näbauer M, Wallwiener D, Kandolf R. High prevalence of viral genomes and inflammation in peripartum cardiomyopathy. Am J Obstet Gynecol 2005;193:363-5.

14. Cunningham FG, Pritchard JA, Hankins GD, Anderson PL, Lucas MJ, Armstrong KF. Peripartum heart failure: idiopathic cardiomyopathy or compounding cardiovascular events. Obstet Gynecol 1986;67:157-68.

15. Ansari AA, Fett JD, Carraway RE, Mayne AE, Onlamoon N, Sundstrom JB. Autoimmune mechanisms as the basis for human peripartum cardiomyopathy. Clin Rev Allergy Immunol 2002;23:301-24.

16. James PR. A review of peripartum cardiomyopathy. Int J Clin Pract 2004;58:363-5.

17. Kothari SS. Aetiopathogenesis of peripartum cardiomyopathy: prolactin-selenium interaction? Int J Cardiol 1977;60:111-4.

18. Mielniczuk L, Williams K, Davis DR, Tang AS, Lemery R, Green MS, et al. Frequency of peripartum cardiomyopathy. Am J Cardiol 2006;97:1765-8.

19. Habli M, O’Brien T, Nowack E, Khoury S, Barton JR, Sibai B, et al. Peripartum cardiomyopathy: prognostic factors for long-term maternal outcome. Am J Obstet Gynecol 2008;199:415.e1-5.

20. Pearson GD, Veille JC, Rahimtoola S, Hsia J, Oakley CM, Hosenpud JD, et al. Peripartum cardiomyopathy: National Heart, Lung, and Blood Institute and Office of Rare Diseases (National Institutes of Health) workshop recommendations and review. JAMA 2000;283:1183-8.

21. Ro A, Frishman WH. Peripartum cardiomyopathy. Cardiol Rev 2006;14:35-42.

22. Witlin AG, Mabie WC, Sibai BM. Peripartum cardiomyopathy: an ominous diagnosis. Am J Obstet Gynecol 1997;176:182-8.

23. Lang RM, Lampert MB, Poppas A, et al. En: Elkayam U, Gleicher N, editors. Cardiac problem in pregnancy: diagnosis and management of maternal and fetal disease. 3rd Ed. New York: Wiley-Liss; 1998. p.87.

24. Abbas AE, Lester SJ, Connolly H. Pregnancy and cardiovascular system. Int J Cardiol 2005;98:179-89.

25. Chapa JB, Heiberger HB, Weinert L, Decara J, Lang RM, Hibbard JU. Prognostic value of echocardiography in peripartum cardiomyopathy. Obstet Gynecol 2005;105:1303-8.

26. Witlin AG, Mabie WC, Sibai BM. Peripartum cardiomyopathy: a longitudinal echocardiographic study. Am J Obstet Gynecol 1997;177:1129-32.

27. Rasmusson KD, Stehlik J, Brown RN, Renlund DG, Wagoner LE, Torre-Amione G, et al. Long-term outcomes of cardiac transplantation for peri-partum cardiomyopathy: a multiinstitutional analysis. J Heart Lung Transplant 2007;26:1097-104.

28. Felker GM, Thompson RE, Hare JM, Hruban RH, Clemetson DE, Howard DL, et al. Underlying causes and long-term survival in patients with initially unexplained cardiomyopathy. N Engl J Med 2000;342:1077-84.

29. Fett J, Christie L. Brief communication: outcomes of subsequent pregnancy after peripartum cardiomyopathy: a case series from Haiti. Ann Intern Med 2006;145:30-4.

30. Elkayam U, Tummala PP, Rao K, Akhter MW, Karaalp IS, Wani OR, et al. Maternal and fetal 
outcomes of subsequent pregnancies in women with peripartum cardiomyopathy. N Engl J Med 2001;344:1567-71.

31. Amos A, Jaber WA, Russell SD. Improved outcomes in peripartum cardiomyopathy with contemporary. Am Heart J 2006;152:509-13.
32. Manolio TA, Baughman KL, Rodeheffer R, Pearson TA, Bristow JD, Michels VV, et al. Prevalence and etiology of idiopathic dilated cardiomyopathy (summary of a National Heart, Lung, and Blood Institute Workshop). Am J Cardiol 1992:69:1458-66.

\section{Conflicto de intereses: ninguno declarado.}

